\title{
Nanosecond-Timescale Low Error Switching of In-Plane Magnetic Tunnel Junctions through Dynamic Oersted-Field-Assisted Spin Hall Effect
}

\author{
S. V. Aradhya ${ }^{1}$, G. E. Rowlands ${ }^{1}$, J. Oh ${ }^{1}$, D. C. Ralph $^{1,2}$, R. A. Buhrman ${ }^{1}$ \\ 1. Cornell University, Ithaca, New York 14853, USA \\ 2. Kavli Institute at Cornell, Ithaca, New York 14853, USA
}

\section{Supporting Information}

\section{Table of Contents}

1. Materials and fabrication. ................................................................................................... 2

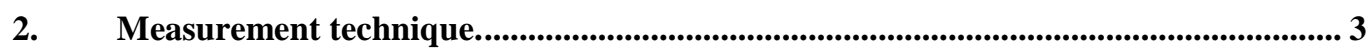

3. Micromagnetic curvature in the free layer.............................................................. 3

4. Magnetic characterization of the free layer....................................................................... 5

5. Switching probability plots in the pulsed regime. …............................................................ 7

6. Comparing Ta and Pt spin-Hall channels........................................................................... 9

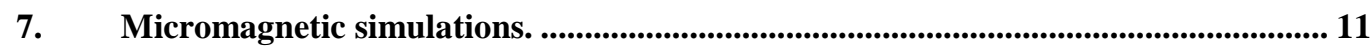

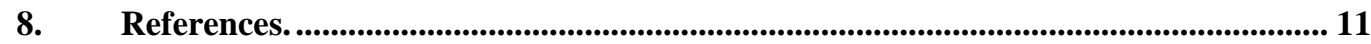




\section{Materials and fabrication.}

The magnetic multilayers are deposited onto thermally oxidized high-resistivity ( $Q>10,000 \Omega \mathrm{cm}$ ) Si wafers using DC and RF magnetron sputtering by Canon ANELVA, Inc. Our multilayers consist of (thicknesses in $\left.\mathrm{nm}, \mathrm{FeCoB} \equiv \mathrm{Fe}_{60} \mathrm{Co}_{20} \mathrm{~B}_{20}\right) \| \mathrm{SiOx}|\mathrm{Ta}(1)| \operatorname{Pt}(5)|\mathrm{Hf}(0.7)| \mathrm{FeCoB}(1.6) \mid$ $\mathrm{MgO}|\operatorname{FeCoB}(1.2)| \mathrm{Ta}(0.2)|\operatorname{FeCoB}(1.2)| \operatorname{FeCo}(1)|\operatorname{Ru}(0.85)| \operatorname{FeCo}(2.5)|\operatorname{IrMn}(7)| \operatorname{Ru}(4)$, where the $\mathrm{FeCoB}(1.6)$ constitutes the magnetic free layer upon which the spin-Hall spin torque acts. The rest of the $\mathrm{FeCoB}$ and $\mathrm{FeCo}$ layers act as a synthetic antiferromagnet and the IrMn layer provides pinning through exchange bias. The nominal Hf insertion layer thickness $t_{\mathrm{Hf}}=0.7 \mathrm{~nm}$ is chosen to reduce the damping of the free layer while maintaining the $\xi_{\mathrm{SH}}$, as reported by Nguyen et $a l^{1}$. We find from flip-chip ferromagnetic resonance measurements on the exchange-biased films, however, a Gilbert damping parameter $\alpha=0.018$ and effective magnetization $M_{\text {eff }}=$ $3.29 \times 10^{5} \mathrm{~A} / \mathrm{m}$ which are quantitatively different from values in Nguyen et al., possibly due to differences in deposition and annealing conditions. The value for $M_{S} t_{F e C O B}^{e f f}$, the product of saturation magnetization and effective thickness of the free layer, is measured to be 0.002 A from vibrating sample magnetometry. From this, we calculate spin-Hall efficiencies in the range $\xi_{S H}^{e f f}$ $=0.052-0.073$ for the three devices in this report, using the macrospin-derived relation ${ }^{2}$ $\xi_{S H}^{e f f}=\frac{2 \mathrm{e}}{\hbar} \mu_{0} M_{S} t_{\mathrm{FeCOB}}^{\text {eff }} \alpha\left(H_{C}+\frac{M_{e f f}}{2}\right) / J_{c 0}$. The multilayer stacks are patterned by deep-UV photolithography (ASML 300C) and etched by $\mathrm{Ar}^{+}$ion milling (IntlVac) into $335 \mathrm{~nm}$ wide, 600 $\mathrm{nm}$ long channels. Using an aligned electron beam lithography (JEOL JBX-6300FS, $100 \mathrm{kV}$ ) exposure and ion milling, we then define the MTJs by fabricating elliptical pillars with three different aspect ratios, as detailed in the main text, in the center of the channels. The ion-milling process is terminated when traces of the channel material become visible in the chamber's secondary ion mass spectrometry endpoint detector. After protecting the devices with electron-beam evaporated $\mathrm{SiO}_{2}$, electrical connections are established to the channel and top contact of the MTJ by means of a liftoff process. The devices are annealed at $360 \mathrm{C}$ for 45 minutes in a vacuum of $<10^{-6}$ Torr, in the presence of a $1.5 \mathrm{kG}$ external field along their long axes. 


\section{Measurement technique.}

For pulse switching experiments, two Picosecond Pulse Labs 10,070A pulse generators are routed through a voltage divider, the capacitive port of a bias-tee, and finally through microwave probes to the device leads. One of the pulse generators is used to apply rectangular switching pulses of varying amplitude $V$ and duration $\tau$ (with 65 ps rise time and 100 ps fall time), while the other is used to apply shallow reset pulses at the $10 \mathrm{~ns}$ maximum pulse duration. As in the $d c$ ramp-rate measurements, $H_{\text {ext }}$ is adjusted to bias the samples at the centers of their magnetic-field hysteresis loops. We emphasize that this field only cancels the average projection of the reference layer's dipole field along the $\mathrm{x}$ direction: according to our micromagnetic simulations, some curvature of the free layer magnetization remains in the $\mathrm{P}$ state though it is most exaggerated in the LA devices. The initial and final MTJ resistance states are measured using a lock-in amplifier connected across a voltage divider formed by the MTJ and a $10 \mathrm{M} \Omega$ series reference resistor.

\section{Micromagnetic curvature in the free layer.}

Through simulations, we find the existance of micromagnetic curvature in both the AP and $\mathrm{P}$ states, due to the influence of the reference layer's residual dipole field, as well as due to edge roughness. Although the AP state appears to have lower curvature than the P state - due to the fact that the dipole field from the reference layer reinforces the shape anisotropy in that case - local curvature can still be expected to exist in both the AP and P states (Fig. 4a,d). Since the anisotropy field is the lowest in the LA device, we can also expect more curvature in the LA devices. This was experimentally observed from the magnetoresistance measurement comparing the three devices (Fig. 1b), where both the AP and P state resistances in the LA device vary with applied magnetic field before and after the switching points. In contrast, the AP states in the MA and HA devices show more stable resistances (Fig. 1b) beyond their respective switching fields. 


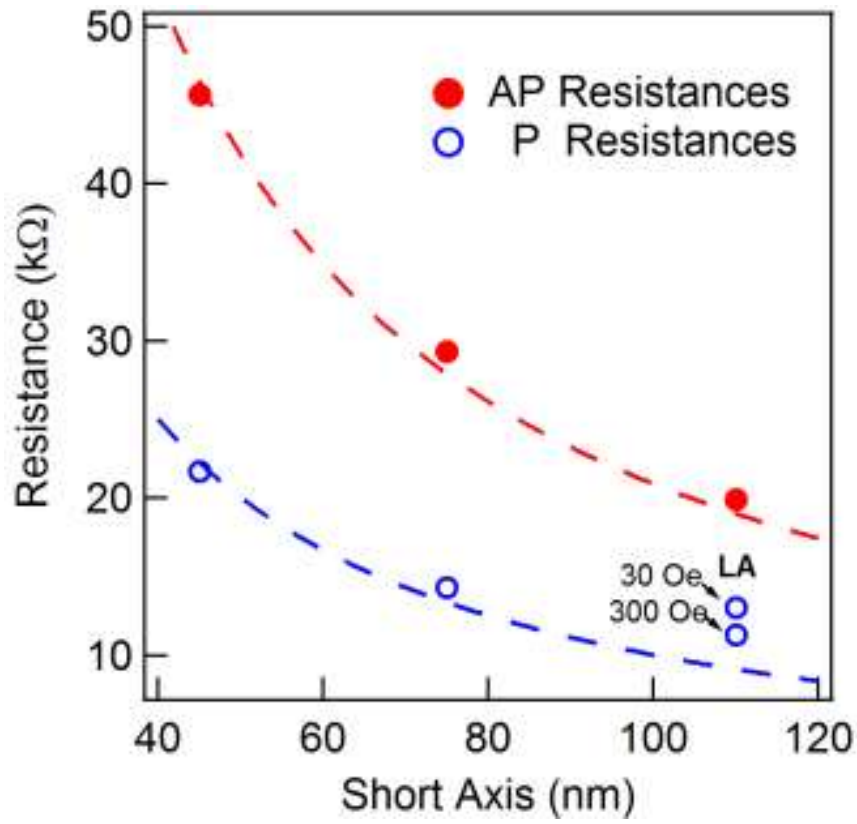

Figure S1. Resistance as a function of MTJ aspect ratio. The P state resistance for the low aspect (LA) ratio device shows deviation from the expected trend based on the device short axis, indicating the existence of significant curvature in the magnetization state. This deviation is reduced when the device resistance is measured at higher fields. Note that the long axis is kept constant at $190 \mathrm{~nm}$ for all three aspect ratios, so the smaller the aspect ratio the larger the area and smaller the resistance of the MTJ. Dashed lines are fits extrapolated from the HA and MA device resistances.

Figure $\mathrm{S} 1$ quantifies the raw $\mathrm{AP}$ and $\mathrm{P}$ state resistances for all three devices, with a $525 \Omega$ contribution from the channel subtracted from the measured total MTJ resistance. Note that the spin-Hall channel contributes half of its total resistance to the measured MTJ resistance, as it forms the 'bottom lead' for the measurement circuit in the three-terminal configuration. The 'top lead' resistance is negligible as it consists of $\sim 50 \mathrm{~nm}$ Ti/Pt material. Using the MA and HA device $\mathrm{AP}$ and $\mathrm{P}$ state resistances as more consistent values for comparison, it is seen that the LA device's $\mathrm{P}$ state resistance is $13.1 \mathrm{k} \Omega$ at $\left(\mathrm{H}_{\text {ext }}-\mathrm{H}_{\text {off }}\right)$ of 30 Oe. $(\sim 43 \%$ higher than the expected 9.1 $\mathrm{k} \Omega$ ). The magnetic origin of this behavior is is apparent in $\mathrm{R}(\mathrm{H})$ loops taken with higher fields, when the $\mathrm{P}$ state resistance goes down to $11.3 \mathrm{k} \Omega$ at $\left(\mathrm{H}_{\text {ext }}-\mathrm{H}_{\text {off }}\right)$ of $300 \mathrm{Oe}$ (Fig. S1). The weak anisotropy in the LA free layer likely increases the curvature in the P state. 


\section{Magnetic characterization of the free layer.}

We use vibrating sample magnetometry (Quantum Design Inc) to measure the magnetic moment of an unpatterned $5 \times 5 \mathrm{~mm}$ die from the same wafer used for fabrication of the devices. Figure S2a shows the moment per area of the free layer as a function of the externally applied magnetic field. The saturated value of $M_{\mathrm{s}} \times t_{\text {eff }}$ is $0.0020 \mathrm{~A}$. Using the as-deposited $1.6 \mathrm{~nm}$ thickness of FeCoB, we can calculate the $M_{\mathrm{s}}$ to be $1.25 \times 10^{6} \mathrm{~A} / \mathrm{m}$. This value does not account for any magnet dead layer at the $\mathrm{Hf} / \mathrm{FeCoB}$ interface.

We use a flip-chip technique to measure the ferromagnetic resonance in an annealed, unpatterned die from the same wafer used to fabricate the devices. Briefly, a microwave waveguide optimized for transmission in the $1-20 \mathrm{GHz}$ range carries a $15 \mathrm{dBm}$ rf power generated by a signal generator (Agilent E8257). The sample is placed on top of this waveguide such that the magnetic layers face the waveguide. A dc magnetic field is scanned using an external electromagnet to detect the resonance condition. This dc field is further modified by using a small ac field generated by Helmholtz coils, which provides an ac signal for lock-in detection. When the resonance condition is satisfied, microwave power is absorbed into the uniform precession mode. The changes in the absorbed power $(\mathrm{d} P / \mathrm{d} H)$ are detected using a rectifying diode, at the ac field modulation frequency.

Figure S2b shows the raw data of $\mathrm{d} P / \mathrm{d} H$ versus the scanned $\mathrm{dc}$ magnetic field. The Lorentzian derivative fit to this data at $9 \mathrm{GHz}$ frequency is also overlaid, and shows very good fidelity to the data. Figure S2c presents the variation of the fitted resonance field, as well as the Kittel model fit

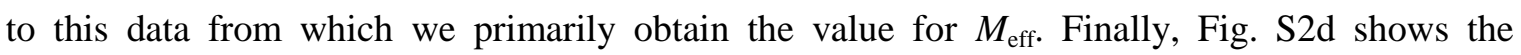
resonance linewidths as a function of the rf frequency, and the linear fit to these points gives us the magnetic damping of the free layer. 

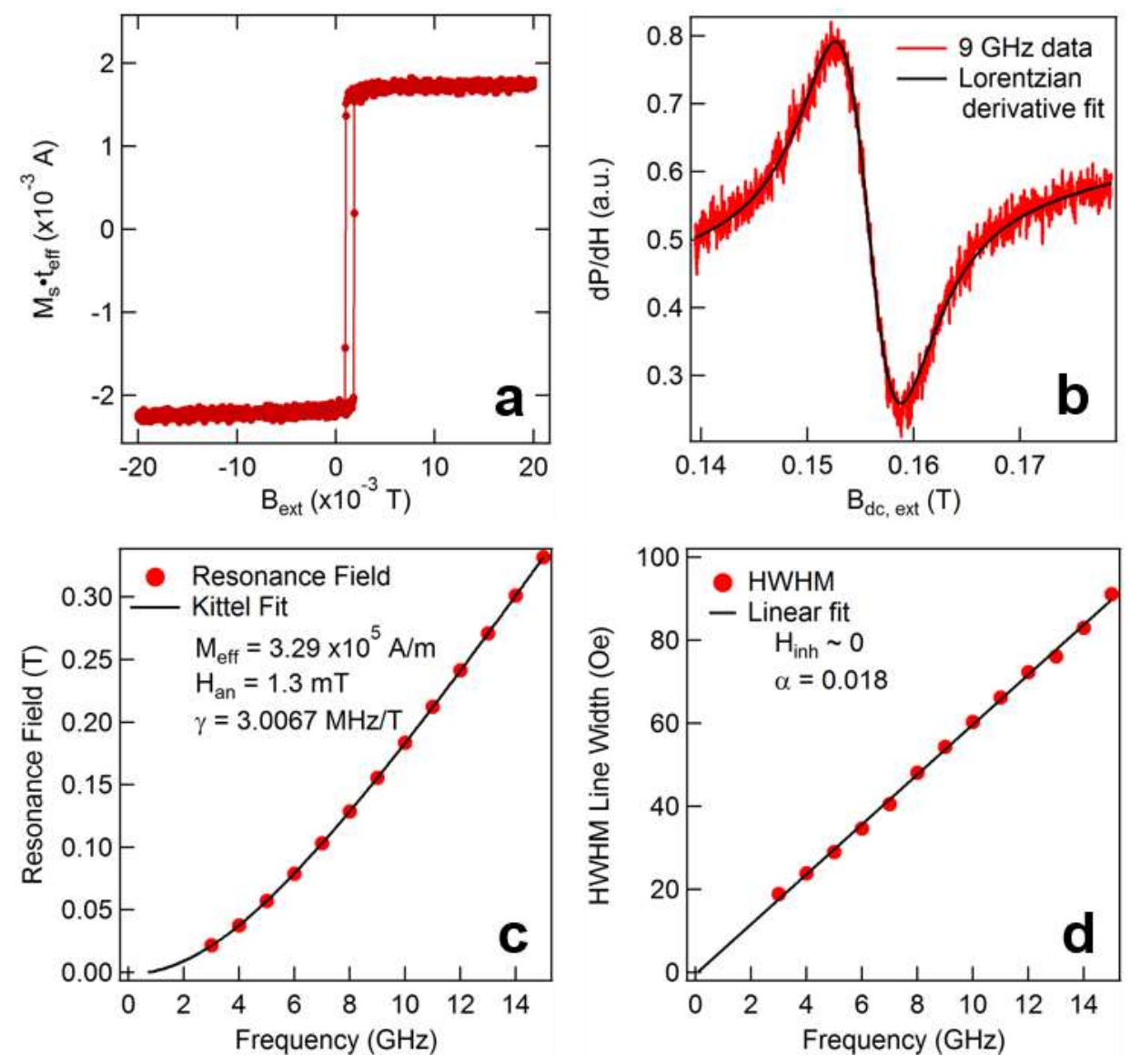

Figure S2. Magnetic characterization of free layer. (a) Magnetic response to an in-plane field measured using vibrating sample magnetometry. (b) A representative ferromagnetic resonance signal from the free layer measured at $9 \mathrm{GHz}$; a Lorentzian derivative fit is overlaid. (c) Fitted resonance fields as a function of applied rf frequency; a Kittel equation fit is overlaid. (d) Half width at half maximum (HWHM) line widths as a function of the rf field; a linear fit is overlaid. 


\section{Switching probability plots in the pulsed regime.}

Pulsed voltage measurements are performed as described in Methods in the main text. The switching probability is measured for AP-to-P and P-to-AP polarities as a function of pulse voltage and pulse duration. For each sweep, the pulse voltage (applied with the appropriate sign for the AP-to-P or P-to-AP polarity) is set using the pulse generator's power attenuator which allows for $1 \mathrm{~dB}$ steps. At each combination of voltage and duration, 200 attempts are made to switch the device and the probability for switching over these attempts is recorded. Figure S3 presents the acquired data for each of the three Pt devices studied in this work. The 50\%

probability switching times (Fig. 2b-d) are then calculated by linear interpolation between the two nearest measured pulse durations that span the $50 \%$ probability crossing, for each pulse voltage used. 

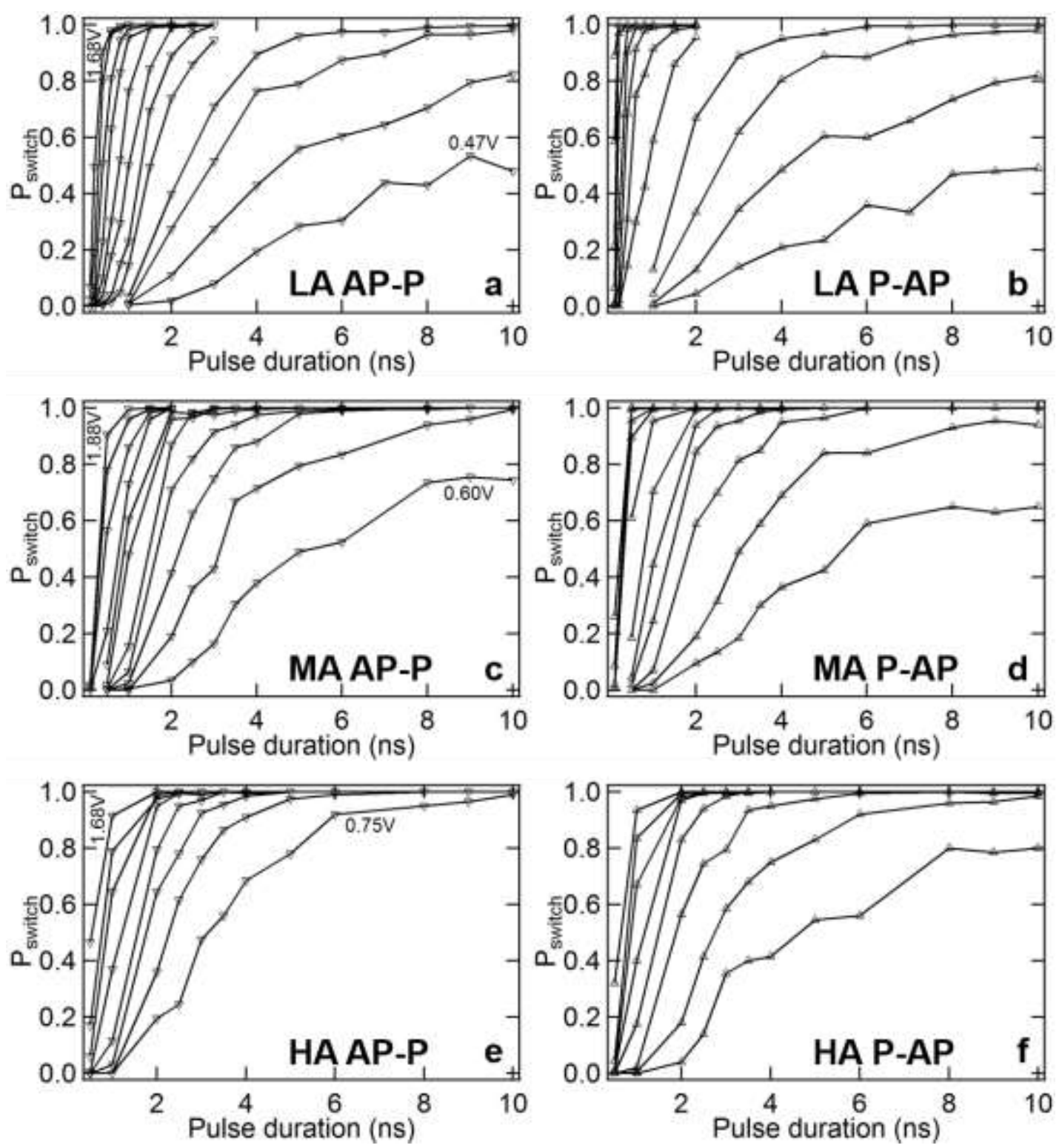

Figure S3. (a, c, e) AP-to-P switching probabilities for LA, MA and HA devices. (b, d, f) P-to-AP switching probabilities for LA, MA and HA devices. The minimum and maximum pulse voltages used are indicated in the plots, with intermediate voltages incrementing in steps of $1 \mathrm{~dB}$ in power. 


\section{Comparing Ta and Pt spin-Hall channels.}

We have also carried out fast pulse switching measurements of a Ta HA three-terminal device. The tantalum materials stack is: $\mathrm{SiOx}|\mathrm{Ta}(7)| \mathrm{FCB}$ (1.8) $|\mathrm{MgO}(1.6)| \mathrm{FCB}$ (3.5) | Ta (4) | Ru (4), with the numbers in parentheses indicating nanometers. The fabrication procedure is identical to the Pt device fabrication detailed in Methods. The HA device dimension is the same as the Pt HA device. The switching probability pulse sweep data is presented in Fig. S4a,b for AP-to-P and P-to-AP switching. Similar to the Pt devices, the 50\% switching time can be fitted well to the macrospin model, as shown in Fig. 4c.
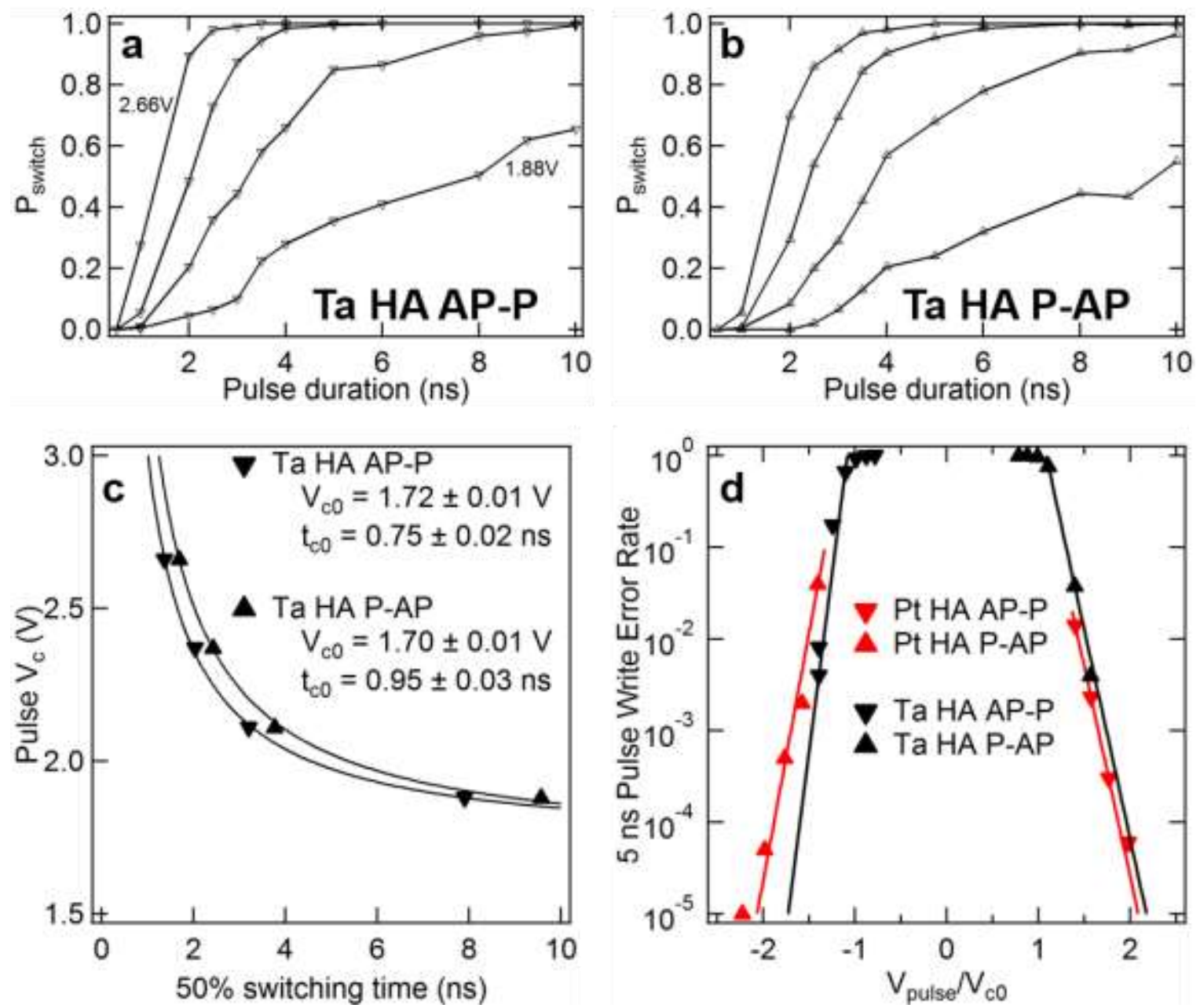

Figure S4. (a) AP-to-P and (b) P-to-AP pulse voltage and pulse duration sweeps. (c) Summary of switching voltage versus 50\% switching times, with macrospin fits overlaid. (d) Comparison of the Ta and Pt HA device 5 ns write error rates as a function of the normalized pulsed voltages. 
We note that due to the different sign of the spin Hall ratio of Ta compared to that $\mathrm{Pt}$ the Oersted field points in the opposite direction for the Ta channel case compared to the Pt channel devices during switching, reinforcing the shape anisotropy. However, we observe that the $t_{\mathrm{c} 0}$ parameter is still very small ( $<1 \mathrm{~ns})$, and comparable to the Pt devices. We do observe a slight asymmetry in the $t_{\mathrm{c} 0}$ values, with the AP-to-P switching being slightly faster than the P-to-AP switching for the Ta case. This is opposite to the Pt case where the P-to-AP switching was slightly faster for the MA and LA devices. Together, these observations indicate that the Oersted field has a more subtle role than just augmenting or diminishing the anisotropy field in the device; the highly favorable switching times appear to be a more general feature of the three terminal device geometry. 


\section{Micromagnetic simulations.}

Simulations are performed using the OOMMF micromagnetic simulation package. ${ }^{3}$ We model the switching response of MA samples, which are given a realistic edge profile (up to the in-plane spatial discretization length of $2.5 \mathrm{~nm}$ ) taken from SEM images of our devices (Fig. S5). We use

an exchange stiffness of $2 \times 10^{-11} \mathrm{~J} / \mathrm{m}$ in the simulations as this value, together with the geometry, reproduces the qualitative shape of the hysteresis curve measured in the experiment. This value is close to that of $\mathrm{Fe},{ }^{4}$ which is in line with the iron-rich composition of the $\mathrm{FeCoB}$ free layer. ${ }^{5} \mathrm{We}$ include both the free and reference layers in the simulation, therefore incorporating their dipolar interactions, and round their thicknesses to the nearest multiple of the $1.5 \mathrm{~nm}$ vertical discretization length. From the equilibrium magnetic state we apply current pulses with 65 ps rise time, including the Oersted field as a uniform magnetic field along the long axis of the sample. All simulations are performed at $0 \mathrm{~K}$. Movies depicting the simulated switching mechanism for both AP-P and P-AP polarities, with and without the Oersted field, for a current density of $2.1 \times 10^{12} \mathrm{~A} / \mathrm{m}^{2}$ are available online as part of the Supporting Information.

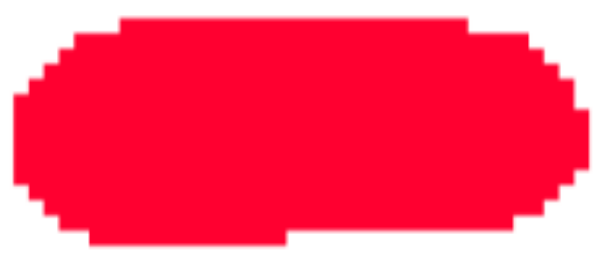

Figure S5. Geometry of simulated free layer, nominally an ellipse of dimension $190 \times 75 \mathrm{~nm}$, corresponding to the MA device in the experiments.

\section{References.}

(1) Nguyen, M.-H.; Nguyen, K. X.; Muller, D. A.; Ralph, D. C.; Buhrman, R. A.; Pai, C.-F. Appl. Phys. Lett. 2015, 106 (22), 222402.

(2) Sun, J. Phys. Rev. B 2000, 62 (1), 570-578.

(3) Donahue, M. J.; Porter, D. G. The OOMMF user guide, Version 1.0; 1999.

(4) Chikazumi, S. Physics of Magnetism; Wiley: New York, 1964.

(5) Yamanouchi, M.; Jander, A.; Dhagat, P.; Ikeda, S.; Matsukura, F.; Ohno, H. IEEE Magn. Lett. 2011, 2, 3000304. 